\title{
Assessing Programmable Logic Controller Skill of Electrical Engineering Student
}

\author{
Puput Wanarti Rusimamto ${ }^{1}$, Ekohariadi ${ }^{2}$, Munoto ${ }^{3}$ \\ Electrical Engineering Department ${ }^{1,3}$ \\ Informatics Engineering Department ${ }^{2}$ \\ Universitas Negeri Surabaya \\ Surabaya, Indonesia \\ puputwanarti@unesa.ac.id
}

\begin{abstract}
Measurements in education can not be separated from the measurement of the students' capabilities of educator candidates. The ability of prospective students can be measured by using a measuring instrument (test). A lecturer should know the scoring theory used to measure the ability of his students. Scoring theory includes classical test theory and modern test theory. Each of these test theories has advantages and disadvantages. Test theory developed in Indonesia today is a classic test theory. Modern test theory has developed in some advanced countries. The progress of information technology has had an impact in the development of modern test theory. The process of calculating the scores of test takers using modern test theory is more complex than using classical tests. In this study, the test items of programmable logic controller (PLC) of electrical engineering students were analyzed using classical test theory approach and grain response theory. The grain difficulty statistics on the classical test theory is the $p$ value, which is calculated based on the proportion of students who correctly answer a test item. Parameter $b$ is a location parameter, indicating the position of the grain characteristic curve in relation to the scale of ability. The correlation between the values of $b$ and $p$ is $-0,980$ of the 38 PLC test items, there are 1 non-fit test items based on the Rasch model. Parameter $b$ has a starting value of $-3,07$ to 4,24 . The parameter of $p$ grain has a value from 0,03 to 0,97 .
\end{abstract} theory

Keywords-assessing PLC, classical test theory, modern test

\section{INTRODUCTION}

There are two kinds of theory about measurement, that is Classic Test Theory and Modern Test Theory. Classical Test Theory is also called Classical True-Score Theory (CTT), called Classical Test Theory because elements of this theory have been developed and applied for a long time, but still survive until now. Modern Test Theory is also called LatentTrait Theory because this theory assumes that the performance of the subject in performing a test can be predicted from latent or persistent ability. Modern Test Theory is also often called the Item Response Theory (IRT), meaning the subject's response to an item indicates its cognitive abilities.

Modern Test Theory appears to address the limitations of Classical Test Theory, that is, the parameters in Classical Test Theory are characteristic of an item dependent on the sample group used to calculate it, besides that Classic Test Theory also requires equality of measurement error for all subjects subjected to the test, the definition of parallel in Classical Test Theory is also very difficult to fulfill in practice. With the presence of Modern Test Theory can answer all these limitations, but keep in mind that Modern Test Theory is not practical, from all the limitations of Classical Test Theory need to be seen also the advantages of Classical Test Theory that is, Classic Test Theory has been developed since the first so that has succeeded in putting the basic concepts of measurement, in addition the Classical Test Theory also has a high practical value.

The classical test theory has grown widely and has become a major flow among psychologists and educators, as well as other areas of behavioral studies. CTT has a weakness because it is examinee sample dependent and sample dependent items [7][8][9]. This weakness triggers a new theory that is more adequate, namely the theory of modern tests, also known as the theory of item response or item response theory (IRT) and also known as the latent traits theory.

Unlike CTTs that focus on information at the test level, IRT mainly focuses on information at the item level so it is expected to cover the shortcomings of CCT. The implementation of the IRT model is based on several postulate assumptions: (1) a participant's performance on an item can be predicted by a set of factors called traits, latent traits, or capabilities; and (2) the relationship between the participants' performance on an item and an underlying latent ability can be described by a monotonic appealing function called item characteristic function or item characteristic curve (ICC) [9]. So the ICC is a depiction in the form of a curve that explains the relationship between latent traits and the performance of the subject on an item. [9] states that the assumptions underlying IRT are unidimensional, local independence, and parameter invariant. Meanwhile, [6] mentions that the most important assumptions are: (1) each item has a item characteristic curve (ICC) and (2) local independence.

Based on the background, conducted research on Assessing Programmable Logic Controller Skill of Electrical Engineering Student 


\section{LITERATURE REVIEW}

\section{A. Classical Test Theory}

Theory of measurement, there are 2 kinds, namely Classical Test Theory (CTT) and Modern Test Theory or Latent-Trait Theory. Elements of this classic test theory has been developed and applied for a long time, but still survive until now. The classical test theory is carried out with a conventional quantitative approach to test the reliability and validity of the scale based on the item. The classical test theory is easy in its application as well as a useful enough model in describing how errors in measurement can affect observational scores. The classical test theory assumes that each observed score $(\mathrm{X})$ on the instrument is a combination of the true underlying score (T) on the error score (E). The equation is as follows:

$$
\mathrm{X}=\mathrm{T}+\mathrm{E}
$$

The thing to note is that the raw score $(\mathrm{X})$ is the only real score; whereas pure score (T) and error score (E) are latent, can not be observed directly. Information obtained from the observation score can be used to test the consistency of the test (reliability). Another assumption to note is that the error score (E) in CTT is random and does not correlate with X or $\mathrm{T}$, and the approximate value is 0 (zero).

Based on the above assumptions, the variant of the raw score is a combination of pure score variant and error score: $\operatorname{Var}(X)=\operatorname{Var}(T)+\operatorname{Var}(E)$. Since the values of $T$ and $E$ are hidden and searched in one test are the individual pure scores (T), the assumptions set forth in the error score (E), get us the test reliability equations: $\mathrm{R}=\operatorname{Var}(\mathrm{T} / \mathrm{Var}(\mathrm{X})$ ). Since pure scores can not be obtained directly; then another way is used to obtain the value of the reliability, ie through two tests the same concept and quality, the correlation between the results of both tests will show the same value with the reliability of the exam.

\section{B. Test Item Analysis}

Modern test theory is often also called Latent Trait Theory is the subject's performance in a predictable test of latent ability. Or better known as the Item Response Theory (IRT) is the subject's response to items that show cognitive. Excess performance of the subject can be seen with Item Characteristic Curve (ICC). This means that the better the performance of the subject will be more responses (answers on the test items) are correct.

Theoretical elements in modern tests include: (a) items (test items), (b) subjects (responses), (c) content of subject responses. Assumptions in modern tests are as follows: (a) the problem item and ability parameters are invariant. This means that the problem created has a positive correlation with the measured ability, (b) unidimensionality, meaning 1 item measures one ability. This assumption is less proven because basically between item 1 with other complement each other, (c) local independence, meaning the response to an item will not affect other items.

The size or rules used to find out which questions are valid (usable) and where the questions are invalid (unusable). The rules are 3, namely: (a) the power of differentiation of the problem, It means the item of question can be considered good if the item can be used to distinguish between highability subjects from low-ability subjects, (b) level of difficulty problem, if the item is not too difficult and not too easy, (c) happen to answer correctly. This means that the problem item can detect a subject who answered randomly and accidentally true.

IRT aims to overcome the weaknesses found in classical measurements. In the IRT, the true student response opportunities, grammatical characteristics or parameters, and the characteristics or parameters of the test participants are linked through a model formula that must be adhered to by either the test group or the test group [9]. That is, the same grain to different test participants must be subject to the rules of the formula, or the same test participants to different test items must also be adhered to the formula. In this process there is what is called invariance between test items and test participants. In modern measurements, the grain difficulty level is not directly related to the ability of the respondents.

The fundamental difference between classical and modern measurements lies in the invariance of scanning, where modern scanning is invariant (unchanged or fixed) to test items as well as to test participants. According to reference [13] that the invariance of test item parameters through group of test participants is the most important characteristic of IRT. It is generally said that the index of difficulty of the test item as the correct proportion of answers makes it difficult to imagine how the test difficulty index can be invariant to the group of test participants of different levels of ability.

\section{Rasch Model}

Rasch modeling emerged from an analysis performed by Dr. Georg Rasch on his own test results. Dr. Rasch did two tests on grade 4, 5 and 6 elementary school students. He found that grade 6 students made less mistakes than grade 4 and 5 on the same problem. Then he describes the graph to show the results of both tests, and finds that the error of one test corresponds to 1.2 errors in the other test, comparing the same in the three classes tested. This means that the degree of difficulty between the two tests has been obtained. In order for this ratio constant to occur, the probability of answering the problem correctly should be the same when the student's ability is the same as the difficulty level. In other words, students have a 50\% chance to answer correctly when the students' ability is the same as the difficulty level.

The simple and precise idea of examining the results of the examinations and graphics he made Dr. Rasch make a popular statement, that 'the opportunity to solve a problem depends on the ratio between the person's abilities and the degree of difficulty of the matter'. After that Rasch modeling continues to be developed into various branches to date. But the basic principle is the same, that is the probabilistic model defined as: "a person having a greater ability than another person should have the greater probability of solving any item of the type in question, and similarly, one item being more difficult than another means that for any person the probability of solving the second item is the greater one" [1].

\section{Programmable Logic Controller}

Programmable Logic Controller (PLC) is a computer specially designed for use on machines (industry). This computer has been designed for use in industrial 
environments, equipped with special input/output and a programming language for control.

\section{a) Hardware}

PLC is a type of control system that has input device, controller and output device. The equipment connected to the PLC which sends a signal to the PLC is called the input divice. While the controller is to perform the calculation, decision-making, control of the input to be issued in the output. How the PLC works can be explained through the block diagram in Figure 1 below.

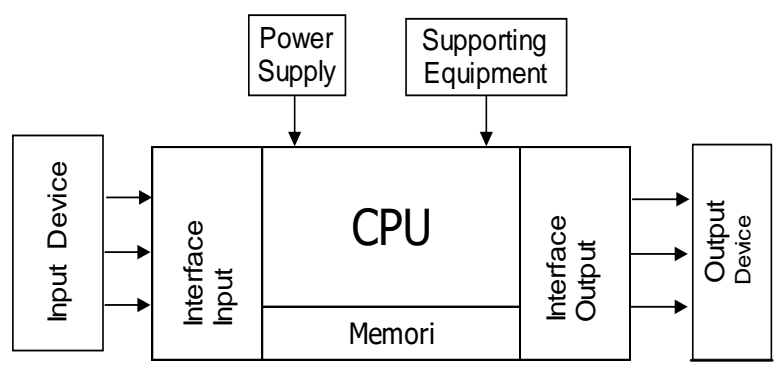

Fig. 1. Block Diagram PLC

From Figure 1 shows that the PLC construction consists of CPU, input device, output device, supporting equipment, and power supply. Explanation of each component as follows:

\section{1) Central Processing Unit (CPU)}

PLC consists of CPU (Central Processing Unit), memory, input interface, and output interface. The CPU is a microprocessor that coordinates the work of the PLC system. It executes programs, processes input/output signals, and communicates with outside equipment.

Memory is the area that stores the operating system and user data. Real operating system software system that coordinates PLC. There are two types of memory: ROM (Read Only Memory) and RAM (Random Access Memory). ROM is a memory that can only be programmed once. Storage program in ROM is permanent, so it is used to save the operating system. There is a type of ROM, EPROM (Erasable Programmable Read Only Memory) whose contents can be removed by irradiating using ultraviolet light and then recharged using PROM Writer. RAM is memory that can be read or writeable, only used to store data temporarily. The data in RAM will be erased if the power supply is lost.

Interface is a circuit used to adjust the signal on the outside equipment. The input interface adjusts the signal from the input device with the signal required for system operation. The output interface adjusts the signal from the PLC with the signal to control the output equipment. The number of available input/output (I/O) terminals depends on the PLC brand. For example OMRON brand PLCs on one unit are available $10,20,30,40$ or $60 \mathrm{I} / \mathrm{O}$ terminals. The number of I/O terminals can be developed by installing an Expansion I O Unit so that it is possible to have $100 \mathrm{I} / \mathrm{O}$.

\section{2) Input Device}

The input device is what gives the signal to the PLC and then the PLC processes the signal to control the output of the device. The types of input devices include:
- Different types of switches, buttons, switch, limit switch, level switch, push switch, proximity switch.

- Various types of sensors, such as light sensors, temperature sensors, level sensors.

- Rotary encoder

- The types of input devices can be seen in Table I.

\section{3) Output Device}

Automation system is incomplete without any output device controlled. The type of output device can be seen in Table I.

The input or sensor unit equipment, controller, and output can be seen in Table I.

TABLE I. EQUIPMENT INPUT, CONTROLLER, AS WELL AS OUTPUT PLC

\begin{tabular}{|l|l|l|}
\hline \multicolumn{1}{|c|}{ INPUT DEVICE } & \multicolumn{1}{|c|}{ CONTROLLER } & \multicolumn{1}{|c|}{$\begin{array}{c}\text { OUTPUT } \\
\text { DEVICE }\end{array}$} \\
\hline $\begin{array}{l}\text { Circuit Breaker } \\
\text { Level Switch }\end{array}$ & Counter & Alarm \\
Motor Starter & Logic Unit & Control Relay \\
Proximity Switch & Timer & Fun \\
Push Button & & Horn \\
Photoelectric Switch & & Light \\
\hline
\end{tabular}

\section{4) Supporting Equipment}

Supporting equipment is the equipment used in the PLC control system, but not part of the system in real terms. That is, this equipment is used for certain purposes that are not related to pegendalian activities. Supporting equipment, among others:

- $\quad$ various types of programmers, ie computers, ladder software, programming console (PC), programmable terminal, and so on.

- Various ladder software, namely: SSS, LSS, Syswin, and CX Programmer.

- Different types of external memory, ie: diskette, $\mathrm{CD}$, flash disk.

- A variety of printing devices in computer systems, such as printers, plotters.

\section{5) Power Supply}

PLC is a digital equipment and every digital equipment requires a DC power supply. This power supply can be supplied from the outside, or from within the PLC itself.

\section{b) Basic Instruction}

Basic instructions that exist in programming using PLC are as follows.

\section{1) $L D($ Load $)$}

LD instruction is required if the sequence on a control system requires only one logical condition and is required to output. Logically like NO relay contacts.

\section{2) $A N D$}

This instruction is required if the sequence of a control system requires more than one logical condition to be fulfilled in order to output an output. Logically like NO relay 
contacts. The AND directive on the ladder diagram is mounted on the series in series (AND logic) in the previous circuit with the corresponding bit.

\section{3) $\mathrm{OR}$}

This instruction is required if the sequence of a control system requires only one of several logical conditions to output an output. Logically like contact NO relay. The OR directive on the ladder diagram is mounted on the circuit in parallel (OR logic).

\section{4) $O U T$}

This instruction function output if all logical conditions of ladder diagram have been fulfilled. Logically like contact $\mathrm{NC}$ relay. So the OUT instruction is used to output the result of a circuit.

\section{5) AND NOT}

This instruction is required if the sequence of a control system requires more than one logical condition to output an output. The logic is like a contact NC relay. The AND NOT instructions on the ladder diagram are mounted on a series in series (AND NOT logic). So this instruction is used to write $\mathrm{NC}$ contacts on the ladder diagram.

\section{6) OR NOT}

Same as the AND NOT instruction, for OR NOT the ladder diagram is mounted on the circuit in parallel (OR NOT logic). So this instruction is used to write NC contacts on the ladder diagram.

\section{7) TIMER (TIM)}

Timer has a limit between 0000 to 9999 in the order of $100 \mathrm{~ms}$. As for the counter has a number order and has a limit between 0000 to 9999 .

\section{8) COUNTER (CNT)}

Timer/Counter on the PLC numbered 512 pieces numbered TC 000 to TC 511. If a number has been used as a timer / counter then the number should not be used again as a timer or counter. So in one program there should be no same timer / counter number.

The timer / counter value of the PLC is countdown from the initial value set by the program. After the countdown reaches zero, the NO timer / counter contact will be ON.

\section{3) Ladder Diagram and Mnemonic Code}

To make it easy to write and insert program on PLC then needed some basic stage. Ladder diagram of a program is made first to facilitate the preparation of mnemonic code. The program form mnemonic code can be directly inserted into the CPU via a PC.

The ladder diagram consists of a line extending downward from the left side with its branches towards the right. The line extending down on the left side is called the busbar. While the branches are called the instruction line. Along the line of instruction are placed conditions leading another instruction line on the next right side. The logic combination of these conditions determines when and how the instructions on the right side are executed.
There are five ways to program PLC: Programming Console (PC).

Prompt Writer.

Sysmac Support Software + Personal Computer.

Factory Intelegent Terminal (FIT).

Graphic Programming Console.

\section{METHOD}

The test is made by the author in order to conduct research Assessing the Skills of Programmable Logic Controller Student of Electrical Engineering. The PLC skills test consists of 40 multiple choice items. The test measures PLC knowledge. The test assesses PLC hardware topics, basic PLC instructions, ladder diagrams, mnemonic code and PLC software. Student responses to the next 40 test items were analyzed using computer program Test Analysis Program [3] and ConQest [17].

Test Analysis Program is used to estimate grain difficulty parameter ( $p$ ) based on classical test theory, while ConQest is used to estimate grain difficulty parameter (b) based on grain response theory.

Performed analysis of PLC instruments. The test results were analyzed using Rasch model response theory. The fit analysis is used to check the unidimensionality of PLC test items. Unidimensionality is a very important assumption on the grain response theory. Test items are unidimensional meaning that the grains measure one ability [10]. One indication of whether the test item is unidimensional is fit data with the Rasch model [16]. To find out whether the Rasch model can predict the response of each respondent, it is used infit mean-square and out-mean-square statistics [16]. STI and CSO statistics are a measure of the degree of compatibility between observational data and the values predicted by the model.

The test items used in this study fit the Rasch model. Selected test items are based on the value of STIs and CSOs. [12] constructed tables to interpret the meaning of the value of STIs and CSOs. Table 2 shows the significance of IMS and CSO values. The test items used have STI and CSO values ranging from 0.5 to 1.5 .

TABLE II. RANGE OF IMS AND OMS VALUE

\begin{tabular}{|l|l|}
\hline \multicolumn{1}{|c|}{ Value } & \multicolumn{1}{c|}{ Application for Measurement } \\
\hline$>2,0$ & Destructive measurement system \\
\hline $1,5-2,0$ & Has no meaning for measurement \\
\hline $0,5-1,5$ & Useful for measurement \\
\hline$<0,5$ & Not beneficial for measurement but not destructive. \\
\hline
\end{tabular}

\section{RESULT AND DISCUSSION}

The test consists of 40 items of choice response (RP) or multiple choice. Responses to 40 test items were then analyzed using the QUEST computer program [1]. There are 2 numbers that the system automatically removes because they are not working. Namely the numbers 35 and 37 , so the total number of test items amounted to 38 . Table 3 shows the estimation results of the selected response type grain parameters. There are 38 grain difficulty parameters (b). 
TABLE III. PARAMETERS OF THE DIFFICULTY OF THE TEST SELECTED RESPONSE TYPE

\begin{tabular}{|c|c|c|c|c|c|}
\hline $\begin{array}{c}\text { No } \\
\text { Butir }\end{array}$ & Par b & Kesukaran & $\begin{array}{c}\text { No } \\
\text { Butir }\end{array}$ & Par b & Kesukaran \\
\hline 1 & 1.2 & 0.36 & 20 & 0.37 & 0.56 \\
\hline 2 & 3.52 & 0.06 & 21 & -0.12 & 0.67 \\
\hline 3 & -3.07 & 0.97 & 22 & 1.75 & 0.25 \\
\hline 4 & -0.4 & 0.72 & 23 & -0.88 & 0.81 \\
\hline 5 & 2.75 & 0.11 & 24 & -1.08 & 0.83 \\
\hline 6 & 1.6 & 0.28 & 25 & 1.91 & 0.22 \\
\hline 7 & 0.25 & 0.58 & 26 & -1.56 & 0.89 \\
\hline 8 & -1.89 & 0.92 & 27 & -0.55 & 0.75 \\
\hline 9 & -0.55 & 0.75 & 28 & 1.6 & 0.28 \\
\hline 10 & 2.75 & 0.11 & 29 & 0.84 & 0.44 \\
\hline 11 & -1.89 & 0.92 & 30 & -3.06 & 0.97 \\
\hline 12 & 1.6 & 0.28 & 31 & -0.88 & 0.81 \\
\hline 13 & 0.84 & 0.44 & 32 & -0.88 & 0.81 \\
\hline 14 & -0.55 & 0.75 & 33 & -1.56 & 0.89 \\
\hline 15 & -1.56 & 0.89 & 34 & 0.25 & 0.58 \\
\hline 16 & -1.81 & 0.89 & 36 & -1.08 & 0.83 \\
\hline 17 & -2.33 & 0.94 & 38 & -0.63 & 0.75 \\
\hline 18 & -0.71 & 0.78 & 39 & -0.04 & 0.64 \\
\hline 19 & 1.6 & 0.28 & 40 & 4.24 & 0.03 \\
\hline
\end{tabular}

The grain difficulty parameter is the point on the scale of ability in which the probability of answering correctly is 0.5 . The parameter is the location parameter, which indicates the position of the grain characteristic curve in relation to the scale of ability. The greater the grain difficulty parameter value, the greater the ability that the test taker needs to obtain a $50 \%$ chance of answering the item correctly. The leftmost curve has a location of -3.07 and the rightmost curve has a location of 4.24. According to a classical test analysis, the grain difficulty has a range from 0.03 to 0.97 .

The relationship between the parameter $b$ and the difficulty of the grains is shown in Figure 2. The correlation between the values of $b$ and $p$ is -0.980 .

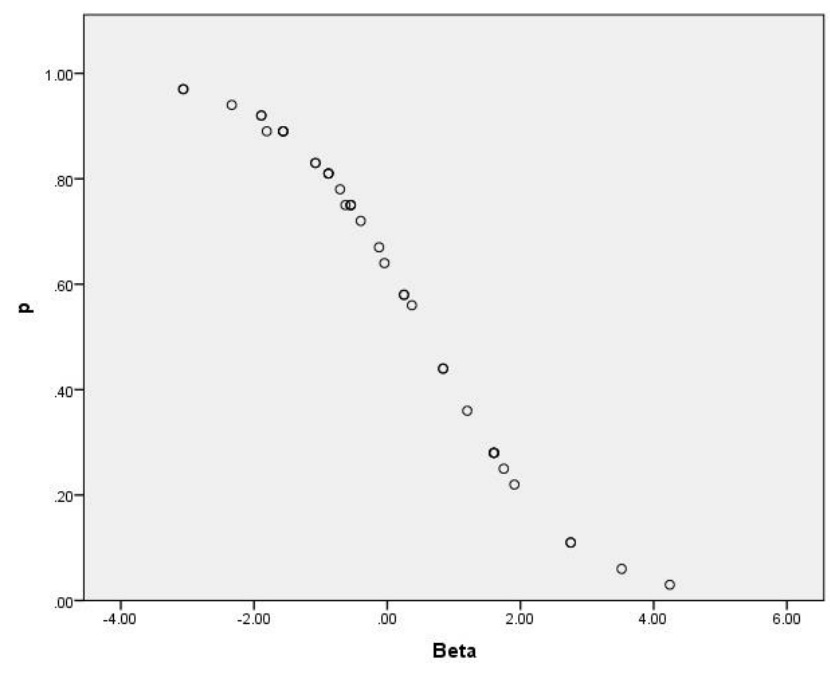

Fig. 2. Relationship between $b$ and $p$

An important characteristic of a set of test items measuring a construct is that they are unidimensional. In Rasch analysis, if all coherent grains form one scale, they are unidimensional. Reference [10] proposed that the grain fit $\mathrm{c}$ is used to examine unidimensionality. Fit grains were analyzed using the Quest [1] program. There are two sizes of fit grains namely Mean-Square lenses (IMS) and Outfit Mean-Square (OMS). The analysis results are shown in Table IV.
TABLE IV. FIT GRAIN ANALYSIS RESUlTS

\begin{tabular}{|c|c|c|c|c|c|}
\hline No Butir & IMS & OMS & No Butir & IM & OMS \\
\hline 1 & 1.02 & 1.02 & 20 & 1.05 & 1.05 \\
\hline 2 & 0.74 & 0.96 & 21 & 0.98 & 0.97 \\
\hline 3 & 0.79 & 1.01 & 22 & 1.17 & 1.08 \\
\hline 4 & 0.96 & 0.98 & 23 & 0.75 & 0.85 \\
\hline 5 & 1.33 & 1.01 & 24 & 0.98 & 1 \\
\hline 6 & 1.36 & 1.17 & 25 & 1.48 & 1.15 \\
\hline 7 & 1.08 & 1.07 & 26 & 0.67 & 0.89 \\
\hline 8 & 1.07 & 1.05 & 27 & 1.01 & 0.99 \\
\hline 9 & 0.95 & 0.96 & 28 & 1.13 & 1.09 \\
\hline 10 & 0.9 & 0.97 & 29 & 1.07 & 1.07 \\
\hline 11 & 0.85 & 0.95 & 30 & 0.43 & 0.95 \\
\hline 12 & 1.16 & 1.08 & 31 & 0.92 & 0.96 \\
\hline 13 & 0.97 & 0.98 & 32 & 0.94 & 0.96 \\
\hline 14 & 1.02 & 1 & 33 & 0.84 & 0.93 \\
\hline 15 & 0.71 & 0.9 & 34 & 1.04 & 1.04 \\
\hline 16 & 0.68 & 0.92 & 36 & 0.79 & 0.88 \\
\hline 17 & 0.56 & 0.93 & 38 & 1.23 & 1.13 \\
\hline 18 & 0.98 & 0.98 & 39 & 1.06 & 1.04 \\
\hline 19 & 0.91 & 0.95 & 40 & 0.77 & 0.98 \\
\hline
\end{tabular}

The value of STI and CSO close to 1.0 indicates a small distortion for the measurement system. A value of 1.0 is the expected value. If the fit observation data with Rasch model then the expectation value is 1.0. The value of STIs and CSOs listed in Table 5 shows that almost all of the test items are useful for measurement. There is no STI value above 1.5. The non-fit items are number 30 which has an STI below 0.44 .

\section{CONCLUSION}

The grain difficulty statistics on classical test theory are $\mathrm{p}$ values, which are calculated based on the proportion of test participants who correctly answer a test item. Parameter $b$ is a location parameter, indicating the position of the grain characteristic curve in relation to the scale of ability. The correlation between the values of $b$ and $p$ is -0.980 . Of the 38 PLC test items, there are 1 non-fit test items based on the Rasch model. Parameter $b$ has a starting value of -3.07 to 4,24 . The parameter of $\mathrm{p}$ grain has a value from 0.03 to 0.97 .

\section{REFERENCES}

[1] Adams, Raymond J \& Khoo, Siek-Toon (1996). Quest : the interactive test analysis system (Version 2.1). Australian Council for Educational Research, Camberwell, Vic

[2] Bond, Trevor., Fox, Christine M. (2015). Applying the Rasch Model Fundamental Measurement in the Human Sciences, Third Edition. New York: Routledge.

[3] Brooks, G.P. (2014). Test Analysis Program (Version 14.7.4)

[4] Cappelleri, Joseph C., Lundy, J. Jason., Hays, Ron D. (2014). Overview of Classical Test Theory and Item Response Theory for the Quantitative Assessment of Items in Developing Patient-Reported Outcomes Measures. Clinical Therapeutics. http://dx.doi.org/10.1016/j.clinthera. 2014.04.006.

[5] Crocker, Linda., Aigina, James. (2008) Introduction to Classical and Modern Test Theory. USA: Cengage Learning. Nelson Education, Ltd.

[6] Embretson SE, Reise SP. (2000). Item Response Theory for Psychologists. Mahwah, NJ: Erlbaum

[7] Fan, Xitao. (1998). Item response theory and classical test theory: an empirical comparison of their item/person statistics. Educational and Psychological Measurement. June 1998 v58 n3 p357 (25). Sage Publications, Inc. 
[8] Hambleton, Ronald K., Swaminathan, Hariharan. (1985). Item Response Theory: Principle and Apllication. Springer Netherlands. DOI: 10.1007/978-94-017-1988-9.

[9] Hambleton, Ronald K; Swaminathan, H; dan Jane Rogers, H. 1991. Fundamentals of Item Response Theory. London: SagePublications.

[10] Hulin, C., Drasgow, F., \& Parsons, C. (1983). Item response theory. Homewood, IL: Dow Jones-Irwin.

[11] Keeves, J.P., \& Masters, G.N. (1999). Introduction. In G.N. Masters \& J.P. Keeves (Eds.), Advances in measurement in educational research and assessment (pp. 1-19). Oxford: Pergamon.

[12] Linacre, J . M. (2000) . Item discrimination and infit mean-squares Rasch Measurement Transactions, 14, 743.

[13] Lord, Frederick, M.1990. Aplications of Item Response Theory to Practical Testing Problems. New Jersey: LawrenceErlbaum Associates, Publishers.

[14] Pessen, David W. (1990). Industrial Automation. Circuit Design and Componen. John Wiley \& Son.
[15] Petrillo, Jennifer., Cano, Stefan J., McLeod, Lori D., Coon, Cheryl D. (2015). Using Classical Test Theory, Item Response Theory, and Rasch Measurement Theory to Evaluate Patient-Reported Outcome Measures: A Comparison of Worked Examples. International Society for Pharmacoeconomics and Outcomes Research (ISPOR). http://dx.doi.org/10.1016/j.jval. 2014. 10.005

[16] Smith, Jr., E.V. (2001). Evidence for the reliability of measures and validity of measure interpretation: A Rasch measurement perspective. Journal of Applied Measurement, 2, 281-311.

[17] Wu, Margaret L \& Wu, M. L \& Adams, R. J \& Wilson, M. R \& Australian Council for Educational Research (ACER). (1998). ACER conquest : generalised item response modelling software. ACER Press, Melbourne.

[18] Anonim. (1991). Omron, Mini H-type PCs C2OH, C28H, C4OH, Training Manual. Singapore: Omron Singapore (PTE) LTD.

[19] Anonim. (2004). Omron, Training PLC Basic. Surabaya: PT. Karisma Pandulima Elektronika. 